\title{
Investigation of Vortex Rings for Free Jet and Synthetic Jet at Various Reynolds Numbers and Strouhal Numbers
}

\author{
Jianlong Chang $\mathbb{D}^{1,2}$ Shizhen Zheng, ${ }^{1,2}$ Yang $\mathrm{Du},{ }^{1,2}$ Junjie $\mathrm{Xu}{ }^{1,2}$ Yanchen Liu, ${ }^{1,2}$ \\ Baoquan Guo, ${ }^{1,2}$ Yutian Pan, ${ }^{1,2}$ and Bing Han ${ }^{1}$ \\ ${ }^{1}$ College of Mechatronic Engineering, North University of China, Taiyuan 030051, China \\ ${ }^{2}$ Institute of Military-Civilian Integration and Innovation, North University of China, Taiyuan 030051, China \\ Correspondence should be addressed to Jianlong Chang; changjianlong1989@126.com
}

Received 7 April 2020; Revised 7 July 2020; Accepted 24 July 2020; Published 13 August 2020

Academic Editor: Luca Chiapponi

Copyright (c) 2020 Jianlong Chang et al. This is an open access article distributed under the Creative Commons Attribution License, which permits unrestricted use, distribution, and reproduction in any medium, provided the original work is properly cited.

\begin{abstract}
The behaviors of vortex rings for free jet and synthetic jet with various Reynolds numbers and Strouhal numbers are numerically studied by the $k-\varepsilon$ model. The positions of the leading vortex of free jet are investigated under the three different conditions, and the effect of Strouhal number on the vortex ring is analyzed in detail. The results show that different Reynolds numbers lead to different positions for the shedding of the vortex rings. During the movement of the vortex rings, the symmetry of the vortex ring in synthetic jet retains in good state, and the vortex rings do not break up with well-arranged distributions. The distance between two adjacent vortex rings near the synthetic jet exit has the close link between the Strouhal number yet is independent of the Reynolds numbers.
\end{abstract}

\section{Introduction}

Jets are widely applied in practical engineering such as ocean engineering, aerospace aircraft launch, jet aircraft, power steam pumps, steam turbines, combustion chambers, automatic control of jet components, and other aspects such as jet cutting, civil fire, and agricultural irrigation [1]. Jets at high Reynolds numbers are generally of high energy, while vortex rings are regularly generated in jets with low Reynolds numbers. Thus, there are many complicated flow phenomena along with the jet flow. Due to the extensive application of jets in various industrial technologies, it is of theoretical and engineering application value to investigate the flow of jet.

Free jet is a kind of typical free-shear flows, which exists in static environment. There is a discontinuity between the jet and the surrounding static fluid for the velocity difference between the jet and the ambient fluid. The discontinuity surface becomes unstable after being disturbed, and thus, vortices are generated, which will entrain the surrounding fluid into the jet. At the same time, the vortices constantly move, deform, fuse, and even generate turbulence. The development of the vortices presents an intermittent phenomenon of free jets, i.e., sometimes laminar flow and sometimes turbulent flow, which is especially evident near the jet boundary. Due to the mutual transfer of momentum between free jet and the ambient fluid, the ambient fluid entrained in free jet will acquire momentum and will flow forward with free jet, and then, a certain velocity gradient will be formed on account of the momentum difference between the two. With the continuous development of free jets, the cross section of free jet continues to expand and the flow rate decreases successively. During the development of free jets, the vortices in free jet show some special flow phenomena such as pairing, fusion, and rupture, which play a vital role in the flow characteristics of free jet [2].

Synthetic jet uses reciprocating motion of a piston or a piezoelectric film to blow/suck fluid. A series of vortex rings/ pairs are formed outside the exit of the narrow hole. These vortex rings/pairs merge with each other in the process of expanding along the direction of the jet. Compared with the traditional flow control, there are unique advantages of no 
additional outflow sources for synthetic jet. And the synthetic jet thus has been widely concerned by many researchers [3]. The main difference between free jet and synthetic jet is that, at the velocity near the exit, the flow velocity of free jet is steady, while the synthetic jet is unsteady and changes periodically.

In recent decades, lots of work on free jets in theoretical analysis and experiments has been done. Not only owing to its wide application background but also free jet as a basic flow contains many basic characteristics of laminar and turbulent flows, which is of great help for us to understand the flow mechanism of fluid. Most of the early researches focused on the round-hole free jet for its typical axial symmetry [4].

3D tomographic PIV, POD analysis, and three vortex identification methods were used by Charmiyan et al. [5] to analyze the coherent structure organization of the turbulent jet flow arising from a slot jet impinging a fixed plate. The results showed that the maximum concentration of the vortices in free jet which occurred around the shear layer was because of instabilities. The TLV turbulent flows at different flow rates in an axial flow pump were simulated [6]. $\mathrm{Nu}$ merical and experimental results showed that the tip leakage vortex trajectory in the axial flow pump was affected by the operating conditions and blade loading.

Grinstein and Devore [7] reported results of time-dependent numerical simulation of spatially developing free square jets initialized with a thin square vortex-sheet with slightly rounded corner regions. The simulations showed that the initial development of the square jet was characterized by the dynamics of vortex rings and braid vortices. Wicker and Eaton [8] took an experimental study to determine the effect of an annular jet on the near-field vortex structure and dynamics of an incompressible axisymmetric jet issuing into a quiescent ambient fluid. The result indicated that axial excitation of the annular flow demonstrated a strong coupling between the large-scale structures in the outer layer and the evolution of the inner layer.

The effects of initial momentum thickness and Reynolds number on free jet with circular holes were conveyed by Kim and Choi [9] by LES. It was found that there was a strong link of the flow characteristics of free jets between the initial momentum thickness and Reynolds number. When the initial momentum thickness was small, the vortex ring in free jet flowed forward. The evolution process of free jet flow field was discussed by Stanley et al. [10] by utilizing DNS. It was conferred that the simulation results were in good agreement with the experimental results, and the flow characteristics of the free jet shear layer were presented. Free jet with round hole was numerically simulated by Bogey and Bailly [11] using LES, and investigations on the flow field and kinetic energy characteristics of free jet were carried out. And the influence of filtering and Smagorinsky dynamic model on energy consumption was analyzed. Attention was also paid to the round-hole free jet by Jewkes et al. [12] with large eddy simulation, and a large eddy simulation model with extremely high accuracy was given to tell the effect of jet inlet velocity condition on separation control.
From the investigations of free jets, it can be concluded that the formation, movement, and evolution of vortex rings at the outlet of free jet are the main results of the jet being affected by the surrounding fluid. The vortex persistently entrains the surrounding fluid, and the fusion and annexation between vortex rings further strengthen the mixing of jet with ambient fluid. Therefore, the formation, movement, evolution, and rupture of vortex ring and the interaction between large-scale coherent structure, shear layer, and ambient fluid in free jet nearly constitute all the research contents of the flow characteristics of vortex ring motion.

It is confirmed by Gharib et al. [13] that the formation of the vortex rings for synthetic jet was limited by the Strouhal number (St). The larger the Strouhal number, the smaller the diameter of the vortex core at the exit of synthetic jet. Pack and Seifert [14] conducted an experimental study on the synthetic jet actuator with an expansion section controlling the outlet flow of the circular tube. The experimental results revealed that the direction of the synthetic jet will drastically affect the deflection angle of the mainstream. While the excitation frequency of the synthetic jet actuator made little difference, the mainstream vector responded rapidly to the synthetic jet control.

Yadav and Agrawal [15] investigated the effect of pulsation frequency on the near-field characteristics of a submerged water jet using the technique of dye visualization. The results showed that the initiation and growth of vortices in the shear layer depends on the pulse frequency. For a given Reynolds number and amplitude, the number of vortical structures and their size changed with frequency. The streamwise and spanwise evolutions of finite span synthetic jets were investigated experimentally using PIV by Amitay and Cannelle [16]. Near the orifice the flow was two-dimensional, while farther downstream the vortex pair lines developed into secondary counterrotating structures.

Yadav et al. [17] had taken PIV-based measurements to investigate the flow structure and mixing characteristics of axisymmetric pulsating jets. It was observed that shear layer in pulsating jet oscillated with a lower frequency as compared to the jet frequency, while the amplitude depended on the location in the flow field. Holdeman and Foss [18] had an experimental investigation to the bounded jet. The result indicated that, in the region away from the bounding plates, the vortex loop distortion was similar to that found in rectangular free jets. However, the bounding plates caused an additional production of streamwise vorticity near the plates, which had no counterpart.

Grinstein [19] had simulations of low-aspect ratio and rectangular-free jets with LES. The result indicated that a more disorganized flow regime in the far jet downstream, where the rotational-fluid volume was occupied by a relatively weak vorticity background with strong, slender tubelike lament vortices. Both theoretical analysis and numerical simulations were undertaken to study the parameters that affect the strength of vortex roll-up of synthetic jets by Zhou et al. [20]. Based on the results from a fully developed oscillating laminar pipe flow, the Stokes number was found to play an important role in determining the thickness of the 
Stokes layer inside the orifice and hence the shape of the velocity profile.

The basic types of synthetic jet actuators were introduced by Luo and Xia [21], and the working principle of synthetic jet actuators, the structure, and the unique flow field characteristics of synthetic jet, as well as the characteristics of synthetic jet technology, were summarized. At the end of the manuscript, the main and potential applications of synthetic jet technology were recommended and summed up, and its control mechanisms in various fields were preliminarily reviewed. Ma et al. [22] inspected the impact of synthetic jet on the wall surface of two-dimensional flat flow. And the combined effect of synthetic jet was analyzed, which was obvious, and can clearly change the average shear stress of the wall surface, with the maximum change of about $15 \%$ relative to the case of no jet.

Gaining much ground in engineering techniques, the flow characteristics of vortex ring structures in free jet and synthetic jet are vital to the research on engineering applications. Therefore, based on the $k-\varepsilon$ model, free jet and synthetic jet under different operating conditions and different Reynolds numbers are numerically simulated. In this manuscript, the numerical simulations of free jet and synthetic jet under different Reynolds numbers are mainly divided into two parts: the formation and flow analysis of two-dimensional free jet vortex ring structure; the vortex ring structure and parameters of two-dimensional synthetic jet. By the investigation of the formation and flow characteristics of the vortex rings for two-dimensional free jet and synthetic jet, it is helpful for the foundation of the flow mechanism of the vortex ring.

\section{Flow Parameters, Governing Equations, and Numerical Method}

2.1. Fluid Flow Equations. The flow of an incompressible, viscous, Newtonian fluid in a domain will contain the vortices formed by singularity of the velocity distribution that can be free to behavior in response to flow-induced forces generated by free jet and synthetic jet. The fluid flow is governed by the unsteady incompressible Navier-Stokes equations, which can be written in the following arbitrary Lagrange-Euler (ALE) formulation:

$$
\begin{gathered}
\frac{\partial u_{i}}{\partial x_{i}}=0 \\
\frac{\mathrm{D} u_{i}}{\mathrm{D} t}+c_{j} \frac{\partial u_{i}}{\partial x_{j}}=-\frac{\partial P}{\partial x_{i}}+\frac{1}{\operatorname{Re}} \frac{\partial}{\partial x_{j}}\left(\frac{\partial u_{i}}{\partial x_{j}}\right),
\end{gathered}
$$

where $u_{i}$ stands for the fluid velocity component along the Cartesian coordinate $x_{i}$ direction. $P$ and $t$ denote the pressure and time, respectively. Re stand for the Reynolds number based on the diameter of the exit $D$, and the flow velocity and the kinematic viscosity of the fluid in Navier-Stokes equations can be modified in order to accommodate the given boundary conditions.

\subsection{Flow Parameters}

(a) Geometric parameters: the schematic diagram of different operating conditions is displayed in Figure 1 . The nozzle center of free jet is located on the line $y=0$, and the width is $D=15 \mathrm{~mm}$.

(b) Physical parameters: the situation pored over here is the injection of fluid into the same fluid. The homogenous fluid is water; therefore, the density of free jet $\rho_{\text {jet }}=1$. The velocity at the entrance of the jet nozzle is evenly distributed, and the kinematic viscosity coefficient of the fluid is 0.001003 . The Reynolds number based on the diameter of the jet nozzle and the maximum velocity of jet varies with the velocity of the jet. The Reynolds number of jet investigated in this manuscript is varying from 100 to 15886.

(c) Structure grids are adopted with the number of grids 221600, as shown in Figure 2. Double precision, simple solution method is employed. The pressure is in standard discrete format, and the remaining physical quantity discrete format is second-order windward format. For the numerical calculation of the low Reynolds number, a good initial condition is calculated using the low Reynolds number $k-\varepsilon$ model.

2.3. Numerical Method. The low Reynolds number $k-\varepsilon$ model is adopted in this manuscript. The term "low Reynolds number" does not refer to the flow within the global scope but refers to the area where the viscous effect dominates. As a result, this model is particularly suitable for detailed characterization of the vortex rings. The low Reynolds number $k-\varepsilon$ model is similar to the standard $k-\varepsilon$ model, but wall functions are not used in the former model. It solves the flow of each position and is a reasonable complement to the standard $k-\varepsilon$ model. The low Reynolds number $k-\varepsilon$ model is with the same advantages as the latter, but with more memory usage. Since a wall function is not demonstrated in, it can simulate lift and drag, as well as heat flux with a higher accuracy. One way to use the low Reynolds number $k-\mathcal{E}$ model is to employ the automatic wall processing function. Firstly, the wall function using the roughened boundary layer mesh is obtained, and then, the boundary layer at the desired wall is refined to acquire a low Reynolds number model [23].

For the low Reynolds number $k-\varepsilon$ turbulence model, the governing equations are as follows:

$$
\begin{aligned}
\frac{\partial(\rho k)}{\partial t}+\frac{\partial}{\partial x_{j}}\left[\rho k u_{j}-\left(\mu+\frac{\mu_{t}}{\sigma_{k}}\right) \frac{\partial k}{\partial x_{j}}\right] & =P-\rho \varepsilon-\rho D, \\
\frac{\partial(\rho \varepsilon)}{\partial t}+\frac{\partial}{\partial x_{j}}\left[\rho \varepsilon u_{j}-\left(\mu+\frac{\mu_{t}}{\sigma_{\varepsilon}}\right) \frac{\partial \varepsilon}{\partial x_{j}}\right] & =\left(C_{\varepsilon 1} f_{1} P-C_{\varepsilon 2} f_{2} \rho \varepsilon\right) \frac{\varepsilon}{k}+\rho E, \\
\mu_{t} & =C_{\mu} f \frac{k_{\mu}^{2}}{\varepsilon}, \\
P & =\tau_{i j}^{\text {turb } \frac{\partial u_{i}}{\partial x_{j}},}
\end{aligned}
$$




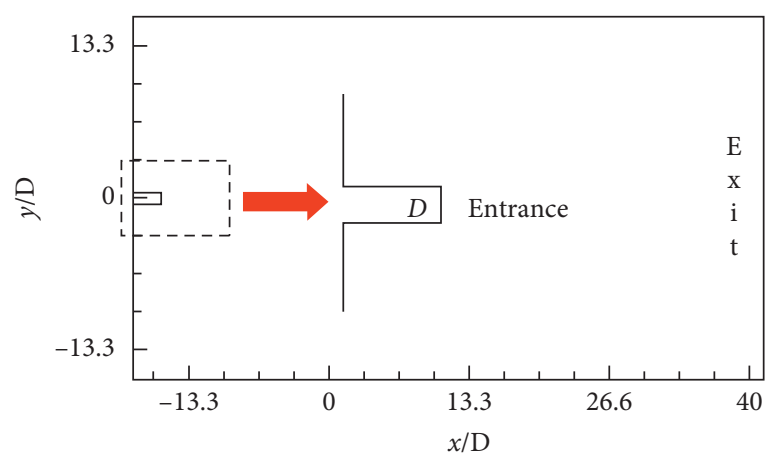

(a)

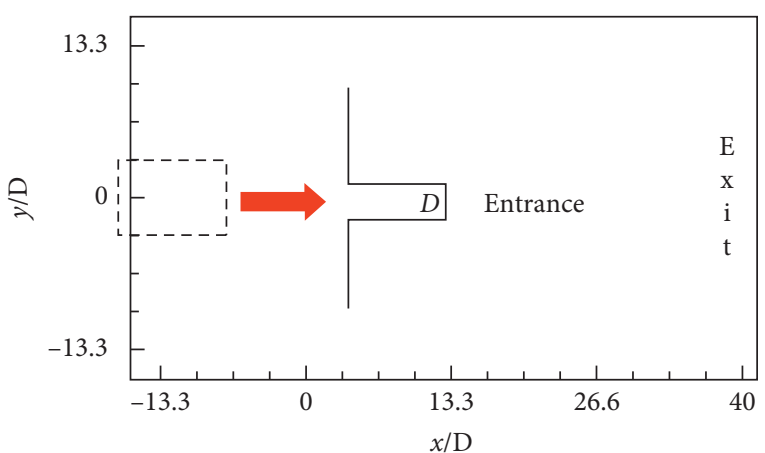

(b)

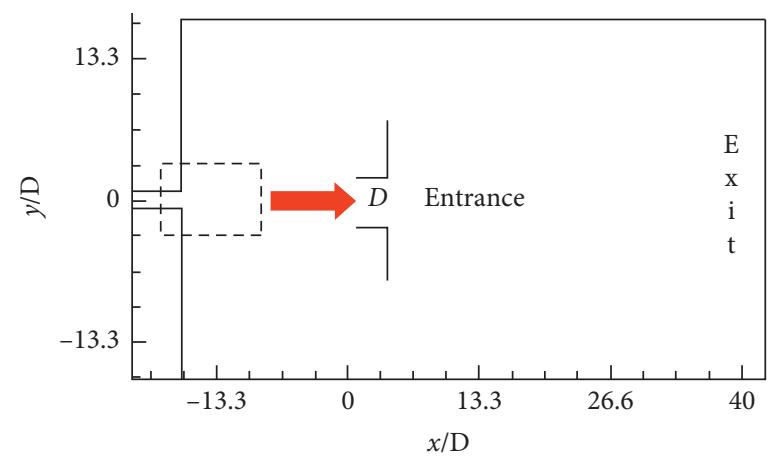

(c)

FIGURE 1: Diagram of the computational domain under different operating conditions: (a) operating condition of rectangular ambulatory plane; (b) condition of nontube; (c) condition of tube.

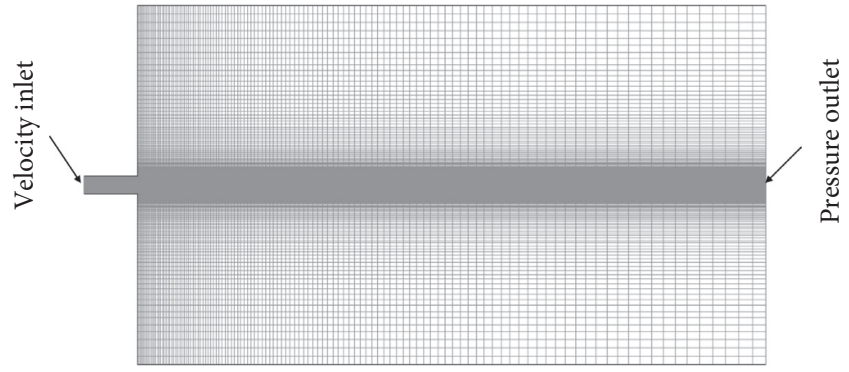

FIgURE 2: Grid distribution in the computational domain.

where $C_{\varepsilon 1}, C_{\varepsilon 2}, C_{\mu}, \sigma_{k}$, and $\sigma_{\varepsilon}$ are model constants. The damping functions $f_{\mu}, f_{1}$, and $f_{2}$ and the extrasource terms $D$ and $E$ are only active close to solid walls and make it possible to solve $k$ and $\varepsilon$ down to the viscous sublayer. In the simulation, the values of $\mathrm{C} 1$ and $\mathrm{C} 2$ are 1.44 and 1.92, respectively. TKE Prandtl number is 1 , and the corresponding TDRE Prandtl number is 1.3 [23].

2.4. Grid Sensitivity Test and Verification of the Accuracy. The grid sensitivity is tested, and the corresponding results are displayed in Figure 3. We have chosen four different grid types $(A * B)$ for the numerical simulation. $\mathrm{A}$ and $\mathrm{B}$ are the nodes in the streamwise and spanwise direction, respectively. As shown in Figure 3, it is found that the deviation of the studied grid types is within 3\%. Subsequently, $508 * 436$ type is adopted in the following simulations.
What follows is the verification of the accuracy of the calculation method and the model adopted in the paper. For this, two representative comparisons in the current research are conducted, and they are (1) Re 2400 (in this manuscript) and $\mathrm{Re} 2358$ (in Ai et al. [24] results); (2) Re 4700 (in this manuscript) and $\operatorname{Re} 4716$ (in Ai et al. results). The comparisons are focused on the penetration of head vortex with time. In Figure 4, $X$ stands for the flow time, and $Y$ stands for the distance of the penetration. As shown in Figure 4, two representative comparisons do not deviate from each other within $5 \%$.

\section{Flow Characteristics of Vortex Rings for Two-Dimensional Free Jet}

3.1. Vortex Ring Structures of Two-Dimensional Free Jet under Different Operating Conditions and the Same Reynolds Number. What demonstrated in Figure 5 is the vorticity field of free jet in the same moment $(t=14 \mathrm{~s})$. It can be acquired that free jet is ejected from the nozzle, and only after a distance of steady flow, the vortex rings are generated. The positions of the leading vortex rings under three conditions including the rectangular ambulatory plane, with and without tube, begin to be, respectively, generated at around the region of $x=-7 D, x=-12 D$, and $x=-8.5 D$. Since the vortex rings of free jet are affected by the instability of Kelvin-Helmholtz, the vortex rings begin to shed from the shear layer and develop along the streamwise direction towards downstream. After the shedding of the leading 


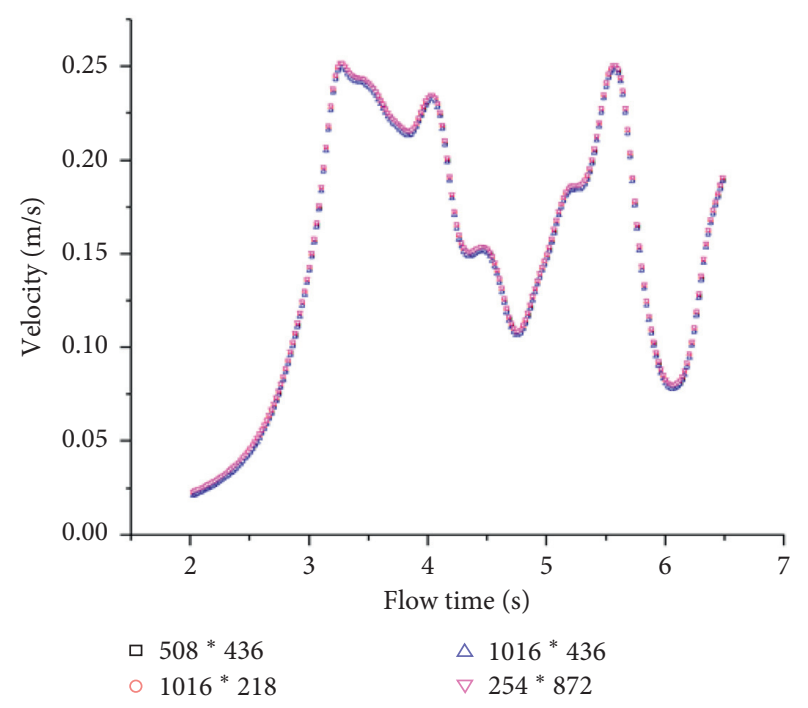

Figure 3: Grid sensitivity test.

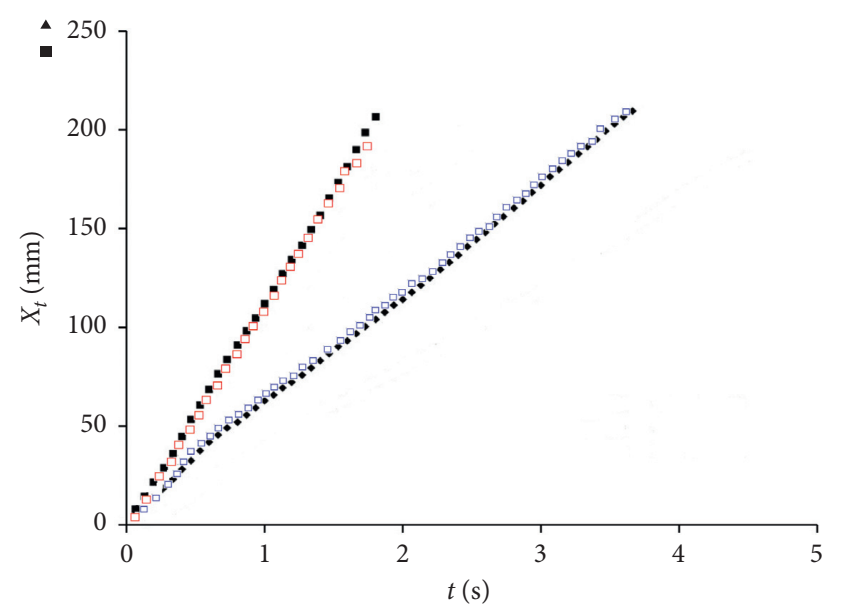

a Re 2400 (in this manuscript)

口 Re 4700 (in this manuscript)

- Re 2358 (in Ai et al.'s results)

- Re 4716 (in Ai et al.'s results)

FIgURE 4: Comparison of results for this manuscript with Ai et al. [24] results in the penetration of head vortex with time.

vortex, the moving velocity of the vortex rings is slower, and the newly generated vortex rings are moving faster. During the development process, the new vortex rings will be merged with the previously vortex rings to reform new vortex rings. Then, the vortices move to the downstream in turn. But in Figure 5, the secondary vortex behind the leading vortex under every operating condition has been in turbulence. The vortex rings begin to manifest asymmetry, and an oscillating wake similar to the Kamen vortex street from the place where free jet vortex rings are shedding is formed.

3.2. Vortex Ring Structures of the Two-Dimensional Free Jet under the Same Operating Condition and Different Reynolds Numbers. Since free jet can retain a higher kinetic energy with the tube, the position where the vortex ring generated is also close to the nozzle. Furthermore, the condition with tube conforms more to the actual situation in engineering, and the condition with tube is selected as the investigation condition for the subsequent numerical simulation.

The vorticity fields of free jet at different Reynolds numbers are depicted in Figure 6. Free jet enters into the tube at a certain initial velocity, passes through the tube, and then enters the outer flow field. As a result, a relatively stable leading vortex is generated in the flow field of $\mathrm{Re}=100$ (Figure 6(a)). With the development of free jet, the vortex ring will continuously move along the streamwise direction until the exit of the flow field. The streamlines of free jet are diffusively distributed in a ray-like manner, while the width of the jet is narrow. With the Reynolds number increasing to $\mathrm{Re}=630$, as proved in Figure 6(b), the leading vortex of free jet is already about to shed from the trailing edge due to the instability of the shear layer in the trailing edge jet. When Reynolds number of free jet is 676, apart from leading vortex, the secondary vortex starts to be generated in the flow field. Nevertheless, owing to the small Reynolds number, free jet moves slowly and is being subjected to viscous and frictional forces of the static fluid in the flow field. The secondary vortex not only sheds slower but also involves in the collision with the newly formed secondary vortex in the trailing jet.

As shown in Figures 6(d) and 6(e), when the Reynolds numbers of free jet ascend to $\operatorname{Re}=1100$ and 2400 , the number of vortex rings in free jet goes up significantly. And due to acceleration of the vortex rings, the shedding process of the secondary vortex speeds up. In the end, the secondary vortex in free jet of $\operatorname{Re}=2400$ will be separated from the newly formed secondary vortices in the trailing edge jet. Yet owing to the jumping-through process between the vortex rings, the fusion occurs between the first secondary vortex and the previously formed leading vortex, as well as the adjacent secondary vortex. This process will make the leading vortex rotate. The fusion of the vortex rings causes instability of the vorticity field so that the newly generated vortex rings in free jet become no longer symmetrical.

When the Reynolds numbers of free jet continue to rise to $\mathrm{Re}=4064$ and 5918, the vorticity field of free jet (Figures 6(f) and 6(g)) is similar to the situation at $\mathrm{Re}=2400$. Though what differs is that the vorticity of the vortex ring is facilitated for the time being. The movement of the vortex rings speeds up, and the interaction between the leading vortex and the series of secondary vortex generated later turns to be pronounced more. As the Reynolds number increases, the intensity and velocity of the secondary vortex will also be elevated. And the size of the leading vortex along with the angle of rotation during the fusion of the leading vortex and the secondary vortex will augment as well. However, with speeding up of vortex ring motion, it is similar to that of a series of newly formed secondary vortex in Figure 6(d). The secondary vortex has not yet shed along the direction of free jet motion, and the new secondary vortex has been generated and connected with the former secondary vortex. This characteristic of free jet will be clearer when at higher Reynolds numbers as $\mathrm{Re}=8100$ (Figure 6(h)) and $\mathrm{Re}=15886$ (Figure 6(i)). 


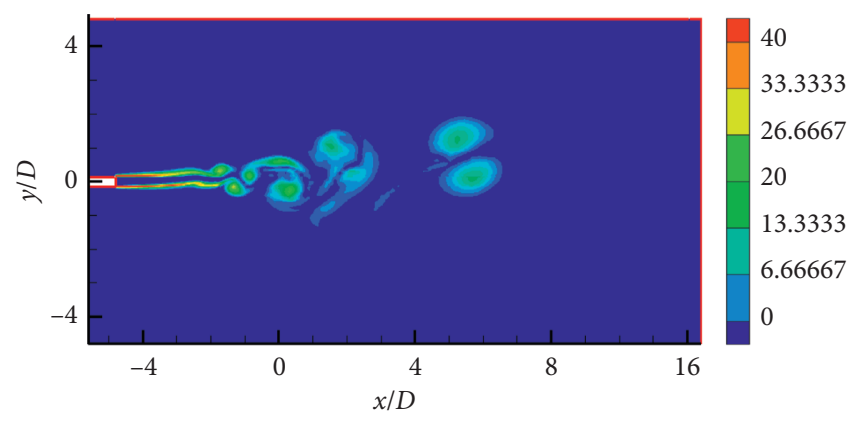

(a)

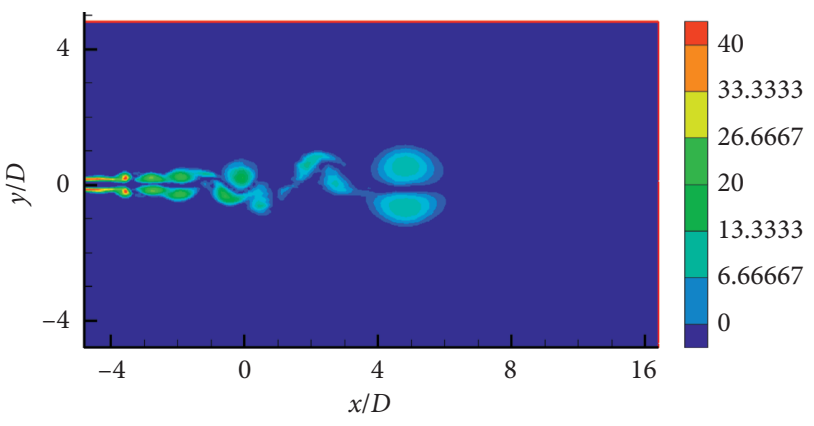

(b)

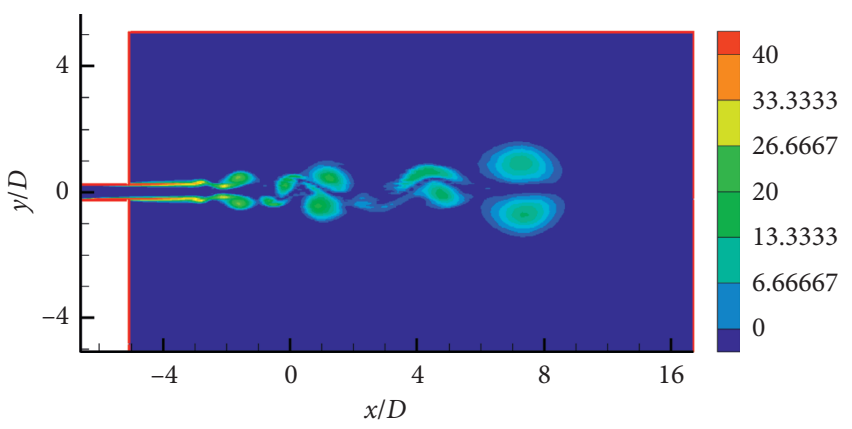

(c)

Figure 5: Vorticity field of free jet at the same time $(t=14 \mathrm{~s})$ : (a) rectangular ambulatory plane condition; (b) no tube condition; (c) condition with tube.

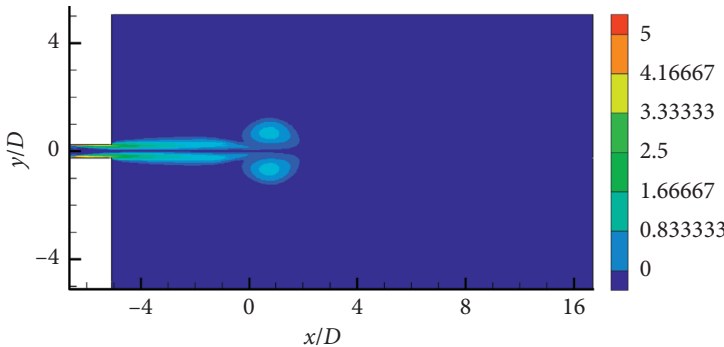

(a)

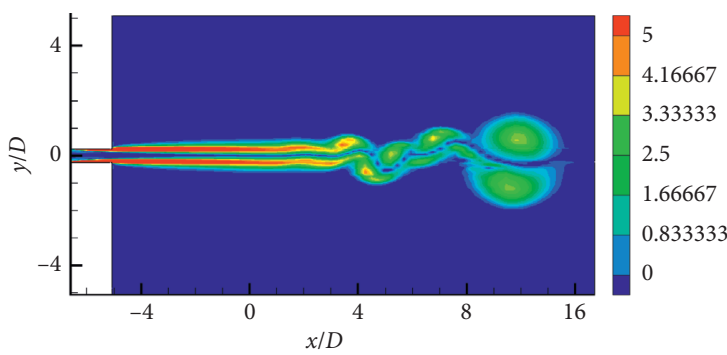

(c)

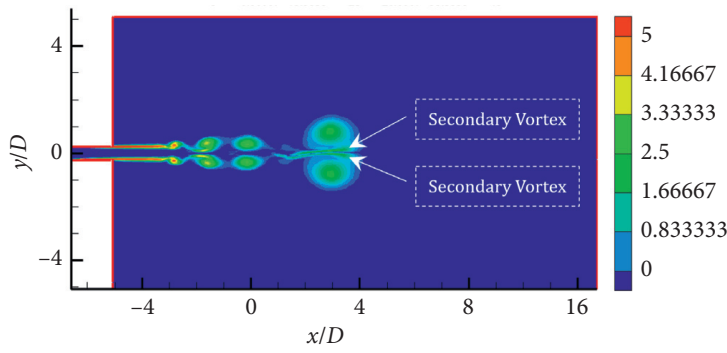

(e)

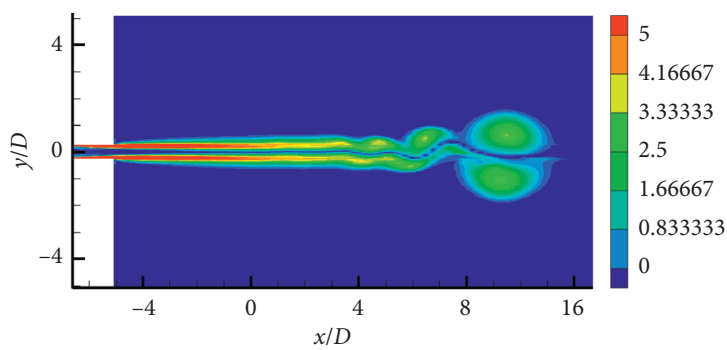

(b)

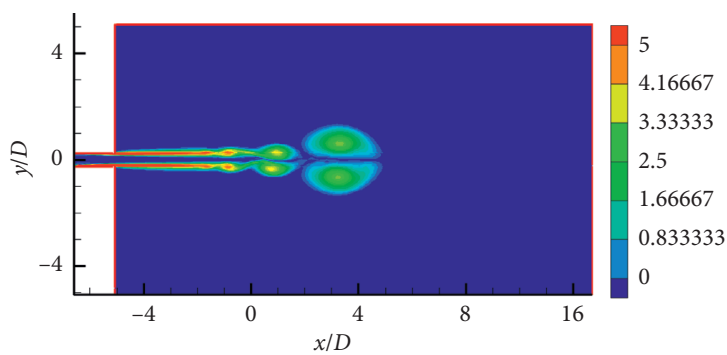

(d)

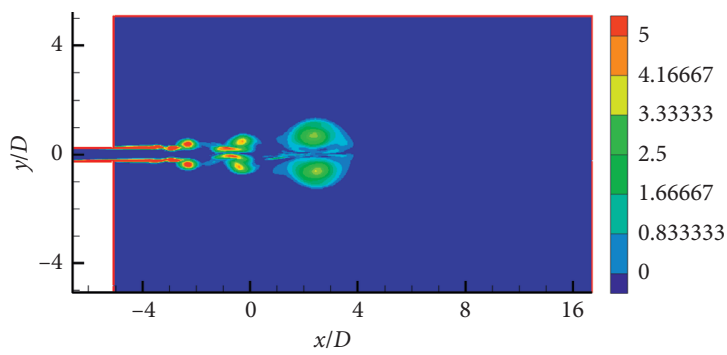

(f)

Figure 6: Continued. 


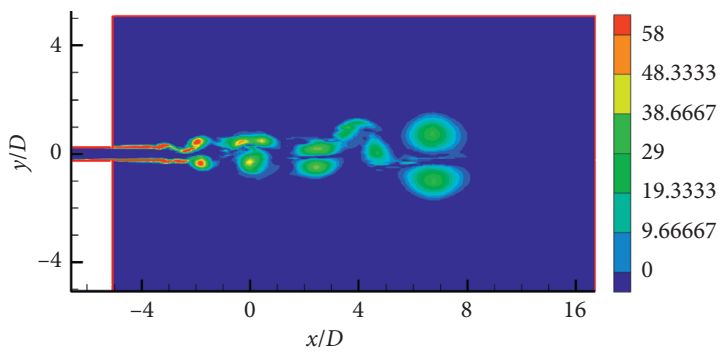

(g)

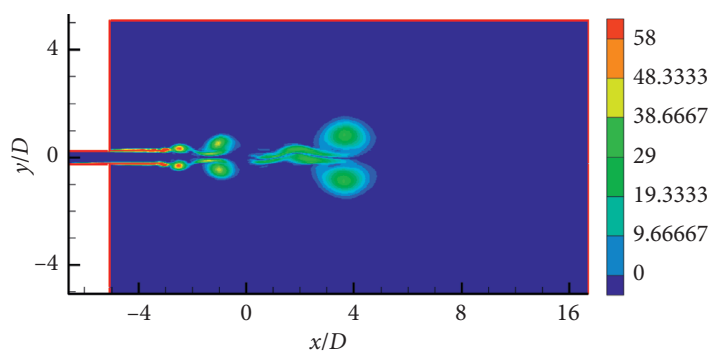

(h)

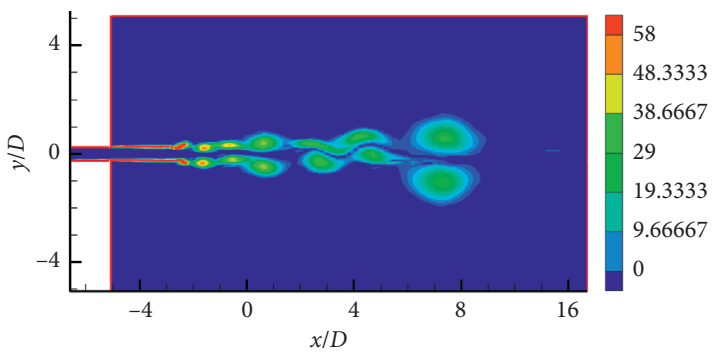

(i)

Figure 6: Vorticity fields at different Reynolds numbers: (a) $\operatorname{Re}=100$; (b) $\operatorname{Re}=630$; (c) $\operatorname{Re}=676$; (d) $\operatorname{Re}=1100 ;$ (e) $\operatorname{Re}=2400$; (f) $\operatorname{Re}=4064$; (g) $\operatorname{Re}=5918$; (h) $\operatorname{Re}=8100$; (i) $\operatorname{Re}=15886$.

When the Reynolds numbers shoot to $\mathrm{Re}=8100$ and $\operatorname{Re}=15886$, the vorticity intensity of free jet is considerably reinforced, and the size of the vortex rings becomes larger. The secondary vortex behind the leading vortices in the vorticity field at two Reynolds numbers becomes more asymmetrical. And there is almost no disconnection between the secondary vortex and the leading vortex and the secondary vortex between one another. The interaction between the vortex rings becomes more noticeable. So the flow characteristics of the unstable free jet will eventually cause instability of the entire flow field after being fully developed, as reflected in Figure 7.

By comparing the vorticity fields of free jet at different Reynolds numbers, the conclusion can be hammered that free jet will generate the leading vortex, which constantly moves along the streamwise direction of the jet. It is from the Reynolds number $\mathrm{Re}=676$ that the secondary vortex begins to appear in the vorticity field. When the Reynolds number is $<676$, there will not emerge shedding of the vortex rings in the vorticity field, and the flow field is relatively stable. When $\operatorname{Re}>676$, the flow field is inclined to lose stability due to the instability of the shear layer, and the shedding of vortex ring is induced. As the Reynolds number rises, the leading vortex and the secondary vortex of free jet will merge together, and the vorticity and velocity of the vortex ring will go up accordingly. The interaction between the secondary vortex and the leading vortex and the secondary vortex between one another will get more marked.

3.3. Evolution of Vortex Rings for Free Jet at $R e=2400$. In addition to the leading vortex in the vorticity field of free jet, there is also a large-scale quasi-ordered structure in the trailing edge jet. The development process of the relevant vortex ring structures in free jet at $\mathrm{Re}=2400$ is verified in Figure 8. It can be seen that, in the formation stage of the leading vortex (Figure 8(a)), the vorticity field of the leading vortex will remain connected with the trailing edge jet. This means that the leading vortex continues to absorb fluid and the vorticity is regularly intensified during the vortex shedding process. In a short period of time later, the leading vortex and the trailing edge jet will separate from each other (Figure $8(\mathrm{~b})$ ). And the shedding time of the leading vortex is about 7-8 times of the formation time of the new vortex ring. In the experimental study completed by Krueger et al. [25] in 2006, it was demonstrated that the leading vortex shedding time was about 9 times of the formation time when the Reynolds number is 1270 . As a result, the velocity of the leading vortex shedding can be inseparable with the Reynolds number.

By conducting the evolution process of vortex rings for the two-dimensional free jet under $\mathrm{Re}=2400$, it can be signified that the separation between the leading vortex and the trailing edge jet is related to the first secondary vortex and the fusion of the secondary vortex ring and the leading vortex. As suggested in the magnified vorticity diagram of Figure 8(d), a smaller concentrated vortex ring (identified by the black arrow) evolves into a new secondary vortex ring. The secondary vortex is actually the initial quasi-order structure in the shear layer of the trailing edge jet, whose size and strength increases with the development of free jet, and gradually ripens into a new secondary vortex. Therefore, the secondary vortex is the direct result of the development and evolution of the unstable layer.

In comparison with the leading vortex, the secondary vortex is of less strength, and it accelerates forward and moves along the leading vortex under the influence of the induced velocity of leading vortex. In the numerical 


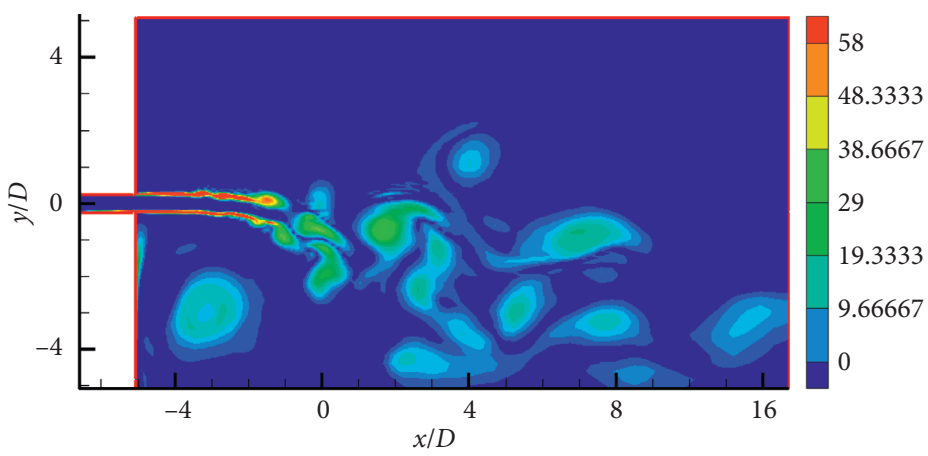

Figure 7: Fully developed vorticity field under $\mathrm{Re}=15886$.

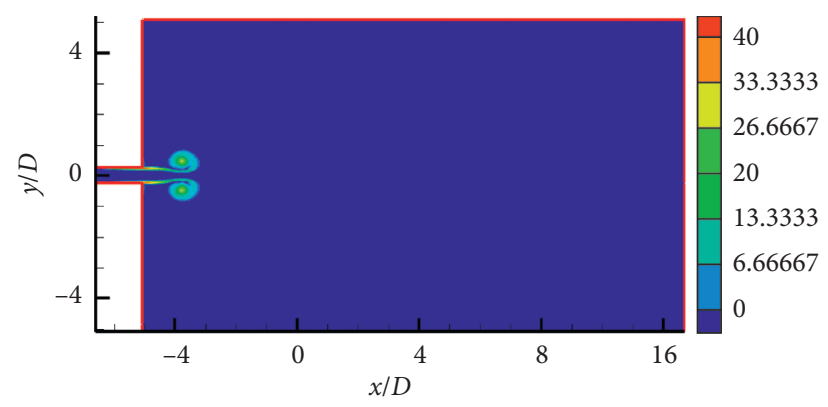

(a)

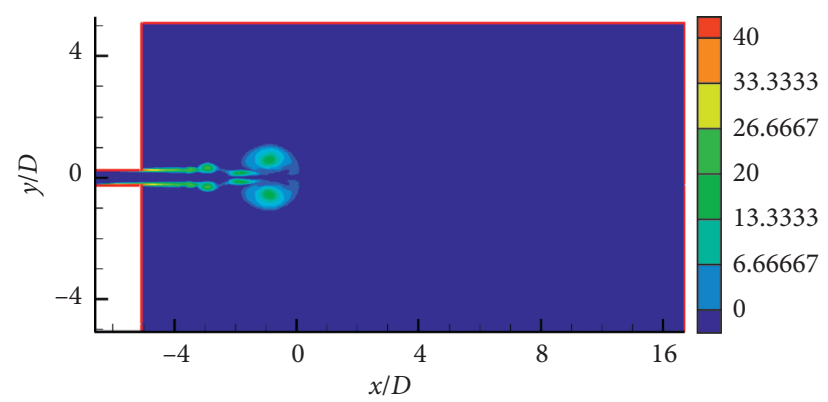

(c)

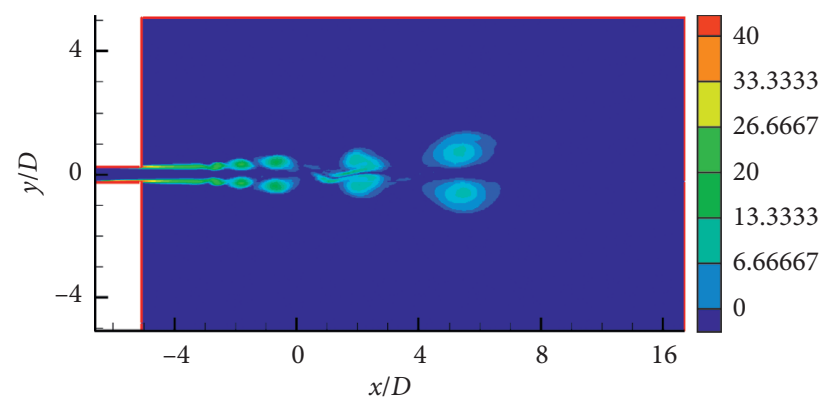

(e)

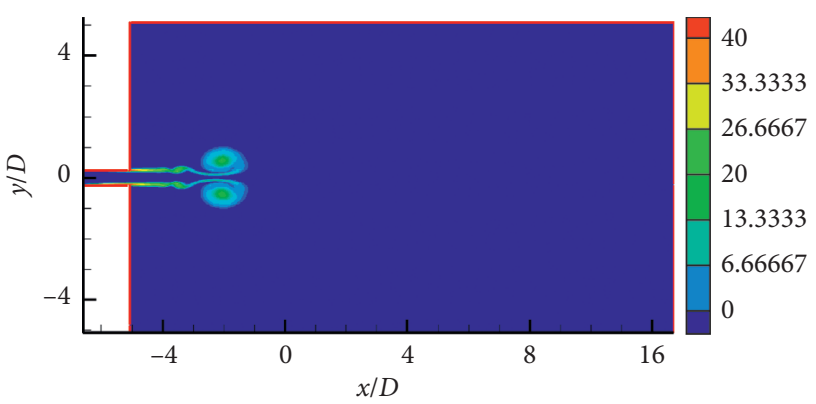

(b)

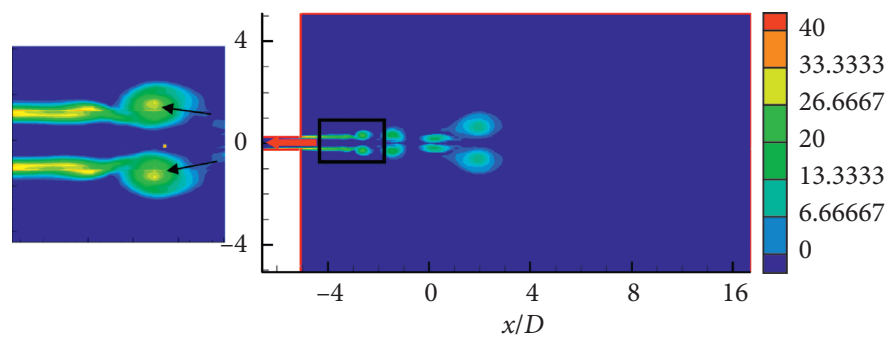

(d)

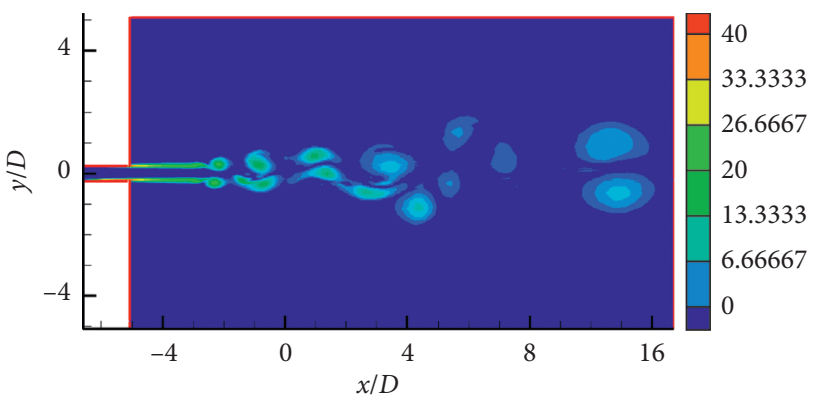

(f)

Figure 8: Evolution of vortex rings for two-dimensional free jet under Re=2400: (a) $t=2.4 \mathrm{~s}$; (b) $t=4.4 \mathrm{~s}$; (c) $t=5.6 \mathrm{~s}$; (d) $t=8.4 \mathrm{~s}$; (e) $t=12 \mathrm{~s}$; (f) $t=18 \mathrm{~s}$.

simulation of free jet at $\operatorname{Re}=2400$, the first secondary vortex does not move beyond the leading vortex; instead, it passes through the gap between the leading vortex pair and then merges into the leading vortex (Figure $8(\mathrm{c})$ ). It is mainly because the intensity of the secondary vortex is smaller than that of the leading vortex, and the result of the fusion of the secondary vortex and the leading vortex is that the secondary vortex generates the front part of the leading vortex. And in turn, the consequence of the first secondary vortex in the trailing edge jet is absorbed by the leading vortex.

The investigation implied in Figure 8 is the continuous variation on the coherent structures in the vorticity field of free jet. However, after the first secondary vortex merged into the leading vortex, the vortex rings can still be observed 
to continue to be fused in the twisted vortex field in Figure 8(e). For the leading vortex, the secondary vortex is a relatively large disturbance, including that the core portion of the leading vortex would redevelop towards steady state. However, in the evolution of vortex rings at $\mathrm{Re}=100$ recorded before, the merged fusion of the vortex ring is not observed before the leading vortex shedding. This infers the shedding of vortex rings and the fusion of the leading vortex and the secondary vortex, and the secondary vortex between one another is closely related to the Reynolds number.

From the results investigated above, it can be confirmed that the fusion process of vortex rings under different Reynolds numbers varies, which is concerned with the evolution of the secondary vortex. The trajectories of the first secondary vortex and the second secondary vortex can be obtained in Figure 8(c), as indicated in Figure 8(c). The first secondary vortex at $\operatorname{Re}=2400$ starts to be rolled up at about $t=4.4 \mathrm{~s}$, and in the position where the leading vortex is formed. The vorticity of trailing edge at different Reynolds numbers under the same operating condition suggests that the reduction of the Reynolds number of free jet delays the generation of the leading vortex and the secondary vortex. When free jet is at a low Reynolds number, the distance between the secondary vortex and the leading vortex increases due to the late formation of the secondary vortex. So the possibility of fusion for the secondary vortex and the leading vortex will also reduce thereafter.

At $R e=2400$, the first secondary vortex in vorticity field is merged into the leading vortex at a later point in the farther location. In view of the dynamics of the secondary vortex, it can be believed that the shedding characteristics of the leading vortex are determined by the formation of the secondary vortex and the interaction between the secondary vortex and the leading vortex, i.e., the mechanism of the separation of the leading vortex and the trailing edge jet. In order to further explain the influence of the trailing edge jet on the development of free jet field, it is necessary to carry out research on the flow characteristics of the trailing edge jet and the shear layer into the leading vortex.

\section{Flow Characteristics of the Vortex Rings for Two-Dimensional Synthetic Jet}

Synthetic jet is an unsteady flow that is produced by a synthetic jet actuator. The understanding of the changes in the synthetic jet over the entire cycle is required, when observing the development and evolution of synthetic jets. The generation process of synthetic jet can be roughly divided into four phases:

(1) The development stage of the synthetic jet is generally considered to be from $t=0$ to $t=0.25 \mathrm{~T}$. At this stage, the synthetic jet is in a blowing state, and the blowing speed is gradually climbed up to the maximum, at which the fluid is sheared. The shearing effect is very strong to generate a pair of vortex rings, which will move forward.
(2) The stable blowing phase of the synthetic jet, which is from $t=0.25 \mathrm{~T}$ to $t=0.5 \mathrm{~T}$, is characterized in that the velocity of the synthetic jet is gradually reduced after being blown out from the outlet. So the shearing effect of the fluid at this stage is weak. The flow field changes slowly.

(3) The inhalation development stage of the synthetic jet, from $t=0.5 \mathrm{~T}$ to $t=0.75 \mathrm{~T}$, is characterized by a progressive increase in the inspiratory velocity, and the velocity direction at the near wall region on both sides of the jet outlet is opposite to the centerline of the synthetic jet, which is inward. And the flow field characteristic of this phase is still in the stage of blowing.

(4) The inspiratory stabilization phase of the synthetic jet is from $t=0.75 \mathrm{~T}$ to $t=1 \mathrm{~T}$, which is characterized by the state of inhalation maintained at the near wall region. But the velocity of inhalation is gradually reduced, the velocity at the center of the jet is extremely small. At this stage, the flow field near the jet exit is barely visible.

The following investigations focus on the flow characteristics of synthetic jet actuator to generate synthetic jet, consequently. Few attempts have been done on the synthetic jet actuator, and the velocity field of the jet is constrained by the dimensionless parameter Strouhal number (St). The Strouhal number is a similarity criterion introduced in the physical similarity and modeling in fluid mechanics and is employed in periodic motion with characteristic frequencies, as follows:

$$
\text { St }=\frac{f \cdot L}{v} \text {. }
$$

4.1. Vortex Ring Structures of Two-Dimensional Synthetic Jet under the Same Operating Condition and Different Reynolds Numbers. It can be concluded from vast varieties of scholars' researches that the synthetic jet with maximum Reynolds number is turbulence, and only a small number of vortex rings is formed at the exit of the synthetic jet. When the Reynolds number is small, stable vortex rings can be formed in the synthetic jet. Therefore, the vorticity field of synthetic jet under the Reynolds number $\mathrm{Re}=100 \sim 15886$ and the Strouhal number $\mathrm{St}=0.22$ is conducted in this section.

Figure 9 is the flow field diagram of the vortex ring structures for two-dimensional synthetic jet under the same Strouhal number $(S t=0.22)$ and different Reynolds numbers. It can be discovered from Figure 9(a) that the flow field structures of synthetic jet are similar to those of free jet flow field at $\operatorname{Re}=100$. Only one vortex pair is formed in the vorticity field of the synthetic jet, and this vortex pair does not shed from the jet. However, what differs from the flow field of free jet is the exit of the synthetic jet, and it is clearly visible that the vortex rings with minimal vorticity is still being generated but will soon be absorbed by the shear layer and jet near the exit. When the Reynolds number is small, the trailing tail of the vortex ring for synthetic jet will not 


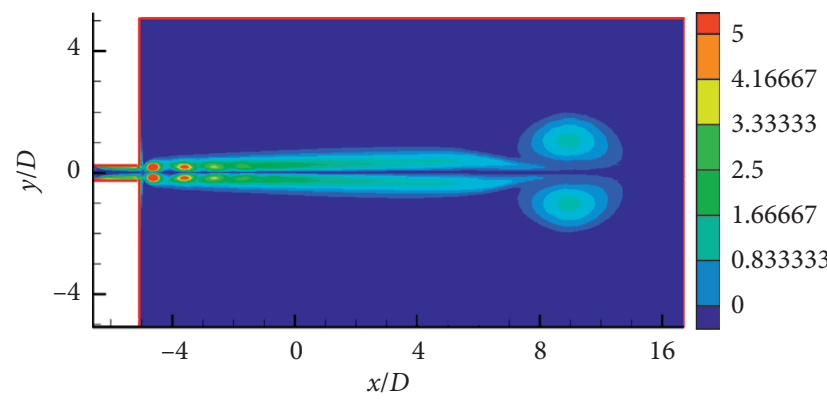

(a)

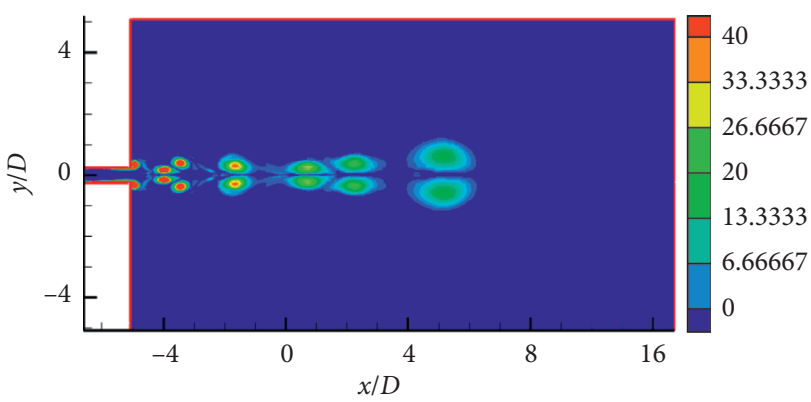

(c)

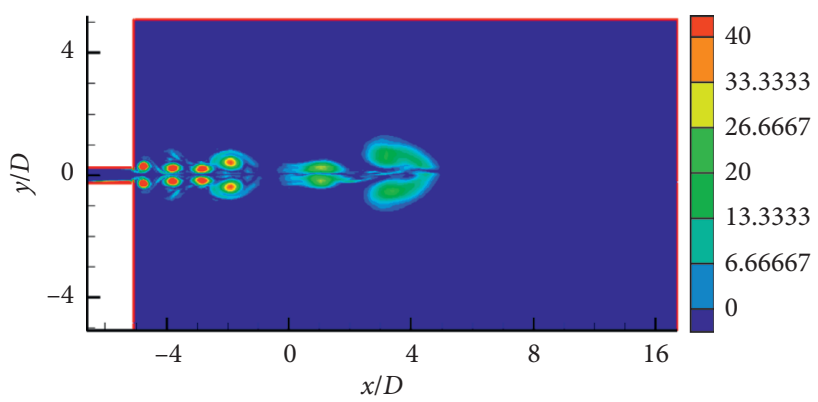

(e)

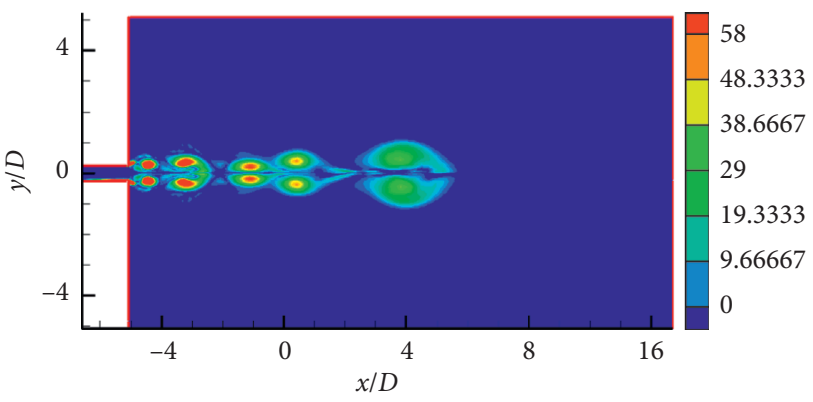

(g)

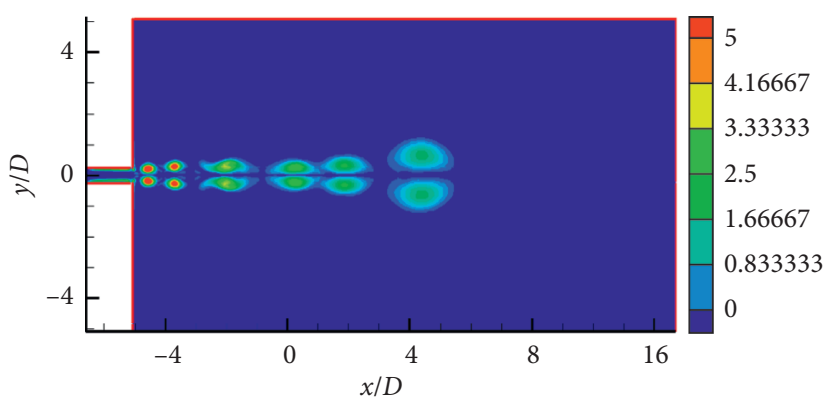

(b)

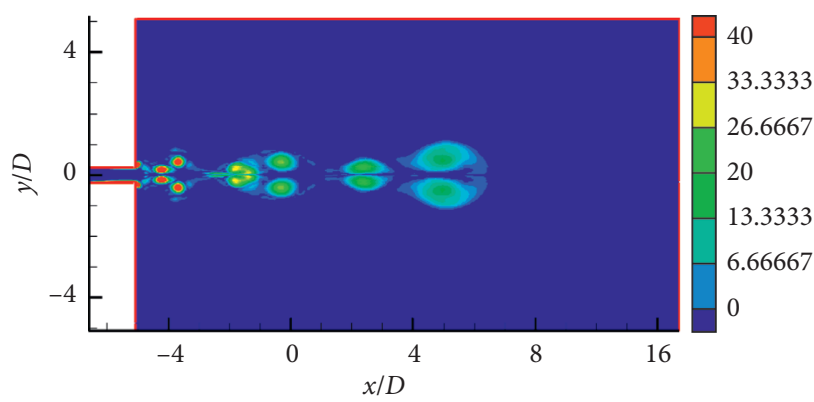

(d)

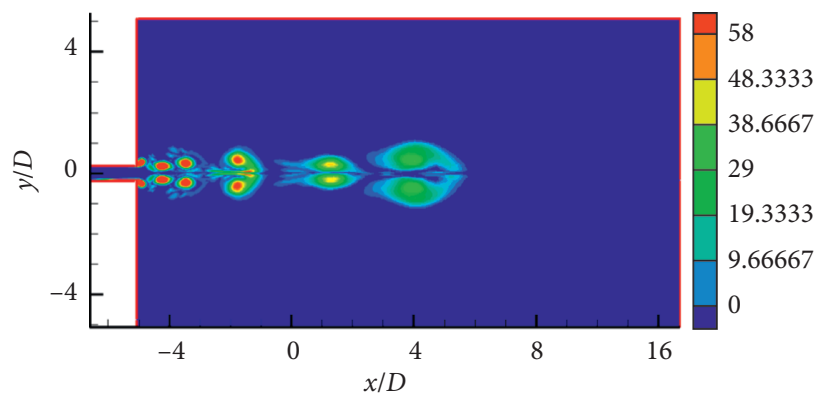

(f)

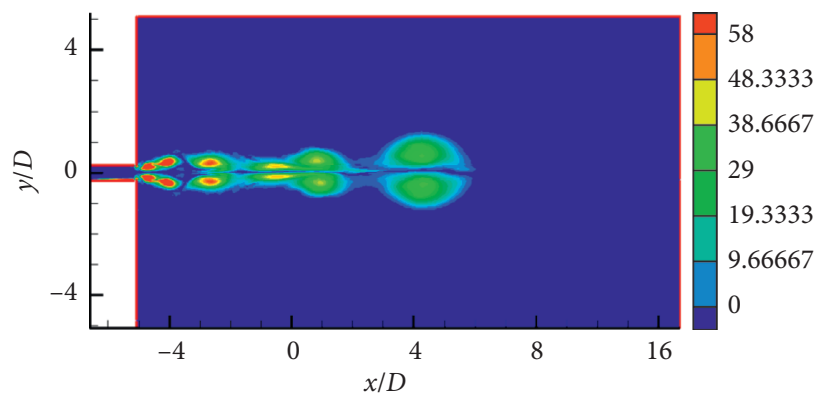

(h)

Figure 9: Vorticity fields of two-dimensional synthetic jet under St $=0.22$ and different Reynolds numbers: (a) $\operatorname{Re}=100 ;(b) \operatorname{Re}=500$; (c) $\operatorname{Re}=1100$; (d) $\operatorname{Re}=2400$; (e) $\operatorname{Re}=4064$; (f) $\operatorname{Re}=5918$; (g) $\operatorname{Re}=8100$; (h) $\operatorname{Re}=15886$.

appear, and it can be seen in the synthetic jet under the large Reynolds number, as a result of the good stability of the vortex ring and less susceptibility to the jet shear layer and fluid at small Reynolds numbers. At a larger Reynolds number, the vortex ring of the synthetic jet is of poor stability and is prone to rupture. The more fluid that is detached from the vortex ring, the more obvious the wake of the synthetic jet.
Starting from the Reynolds number $\operatorname{Re} \geq 500$ (Figures 9(c)-9(h)), a series of vortex rings are generated at the exit of the synthetic jet. Due to the effect of the Strouhal number and the Reynolds number, several vortex rings are generated very quickly at the exit of the synthetic jet, and these vortex rings will soon be fused, forming a large leading vortex that moves forward. Although there are a number of trailing edge vortices behind, fusion and annexation will 
also occur among these vortex rings. But during the movement of the vortex rings towards downstream, the symmetry of the vortex rings remains well, and the vortex rings do not break up and are well arranged. The distance between the vortex ring of the last cycle and the vortex ring generated in the next cycle does not change with the Reynolds number, and it seems to be only associated with the Strouhal number. When the Strouhal number is at a certain value, the distance between the vortex rings is ought to be fixed. Only that the trailing tail of the vortex ring at high Reynolds number is more obvious, and it is hard to distinguish it from the trailing edge vortex in the trailing edge jet for low Reynolds number.

As the Reynolds number of the synthetic jet increases, the vortex rings move downstream along the direction of the synthetic jet. There is a tendency for the vorticity field of the synthetic jet to develop into the turbulent flow, and it is especially apparent in Figure 9(e) $(\mathrm{Re}=4064)$, which shows that the leading vortex of the synthetic jet has been turned into a turbulent flow. There is an effect for the increasing Reynolds number on the fusion, annexation, and fragmentation between the vortex rings. With the adding of the Reynolds number, the turbulent flow is more likely to occur after the synthetic jet is fully developed.

4.2. Vorticity Field of the Synthetic Jet under Different Reynolds Numbers and Different Strouhal Numbers. The impact of the Strouhal number on the synthetic jet accounts for a great proportion; thus, the vorticity field of the synthetic jet under different Strouhal numbers $(\mathrm{St}=0.11,0.22$, and 0.33$)$ and low Reynolds numbers $(\mathrm{Re}=100,1100$, and 2400) are investigated hereby.

As given in Figures 10 and 11, the vorticity field of the synthetic jet at low Reynolds numbers and of Strouhal number $\mathrm{St}=0.11$ and $\mathrm{St}=0.33$ is, respectively, presented, and the vorticity fields of $\mathrm{St}=0.22$ under different Reynolds numbers have been depicted in Figure 9.

Comparing Figures 9(a), 10(a), and 11(a), it can be safely put forward that when $R e=100$, the flow field structures of the synthetic jet at $\mathrm{St}=0.22$ and $\mathrm{St}=0.33$ are almost the same. In the corresponding vorticity field, only one large vortex ring appears, and the vortex ring does not shed. And when the synthetic jet is at $S t=0.11$, the vortex rings are generated continuously in the synthetic jet vorticity field and move stably in the streamwise direction, in virtue of the different Strouhal numbers of the synthetic jet. When the Strouhal number is larger $(\mathrm{St}=0.22, \mathrm{St}=0.33)$, there is short duration of both the synthetic jet velocity field and formation of a vortex ring at the exit. Nonetheless, on account of the effect of the shear layer around the jet exit, combined with the low velocity of the synthetic jet at low Reynolds number, the vortex ring cannot move fast along the synthetic jet being retarded near the exit.
The vortex rings of the synthetic jet are generated faster at a large value of Strouhal number. In the vicinity of the synthetic jet outlet, the vortex rings will continue to be merged and annexed and are under the interaction with the shear layer near the synthetic jet outlet. Finally, a larger vortex ring is formed. When $S t=0.11$, the duration of both the velocity field and formation of the synthetic jet vortex ring gets longer. When a vortex ring is formed at the jet exit, the former vortex ring has moved along the synthetic jet for quite a time. While for the reason that the distance between the newly generated vortex ring and the previously formed vortex ring is large, the interaction between the vortex rings with one another counts for little. Hence, the fusion and annexation of the vortex rings may not happen very quickly.

When the Reynolds number is at $\mathrm{Re}=1100$ and $\operatorname{Re}=2400$, regardless of the value of the Strouhal number, the leading vortex with a large vorticity intensity formed by the fusion of several small vortex rings at the exit of the synthetic jet will move forward. The vortex rings generated in the trailing edge synthetic jet also undergo fusion and annexation. But in the process of the movement towards downstream, the symmetry of the vortex ring remains well and the vortex rings do not break up.

However, when at the same Reynolds number, as the Strouhal number rises, the stability of the far field region of the vorticity field for synthetic jet will continue to decline. When $\operatorname{Re}=2400$ and $\mathrm{St}=0.11$ (shown in Figure 12(a)), after the synthetic jet is fully developed, the vorticity field of the synthetic jet becomes unstable after about $x=D$. And as manifested in Figure 12(b), when $\mathrm{Re}=2400$ and $\mathrm{St}=0.33$, after the synthetic jet vorticity field is fully developed, the vortex ring becomes unstable at $x=-1.5 D$ and turns to be asymmetrical. Under the same Reynolds number, the Strouhal number makes a difference in the distance between the leading vortex and the trailing edge vortex and the distance between the trailing edge vortices. In the end, as the Strouhal number gets larger, the distance between these vortices will be drastically reduced.

The substance displayed in Figure 13 is the vorticity field of synthetic jet under different Reynolds numbers at $\mathrm{St}=0.22$ in near field. The distance between the vortex rings of the last cycle and those generated in the latter cycle does not change with the Reynolds number. It is only concerned with the Strouhal number. When the Strouhal number is constant, the distance is a fixed value. For example, in Figure 13(a), when the Reynolds number is 1100 and Strouhal number is 0.22 , the distance between two adjacent vortex rings near the synthetic jet exit is $d_{1}=2.7995 D$. When the Reynolds number grows to be the value of $\operatorname{Re}=5918$, the distance between adjacent two vortex rings is $d_{2}=2.8002 \mathrm{D}$, and the values of $d_{1}$ and $d_{2}$ are basically the same. As a comparison, when the Reynolds number and Strouhal number are 1100 and $\mathrm{St}=0.11$, respectively, in Figure 13(c), the distance between two 


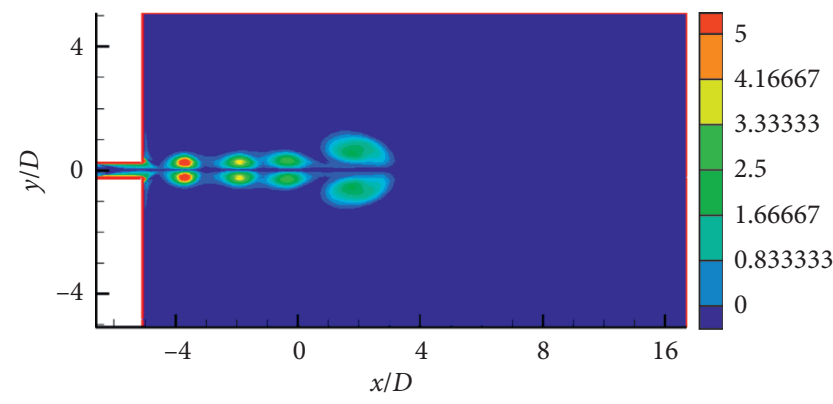

(a)

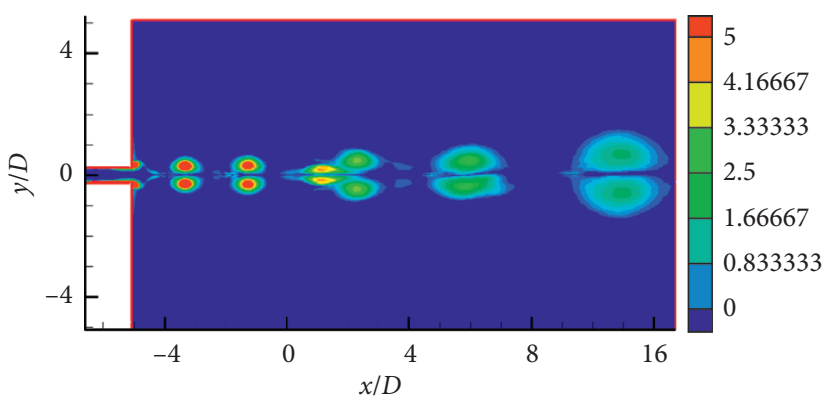

(b)

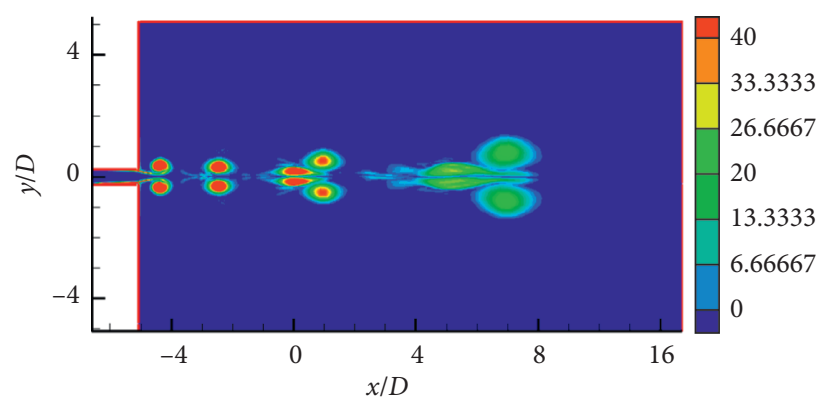

(c)

Figure 10: Vorticity field of synthetic jet at $\mathrm{St}=0.11$ : (a) $\operatorname{Re}=100$; (b) $\operatorname{Re}=1100$; (c) $\operatorname{Re}=2400$.

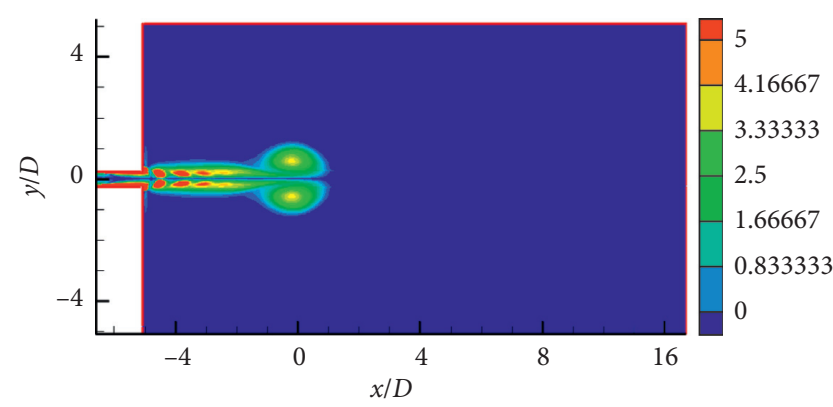

(a)

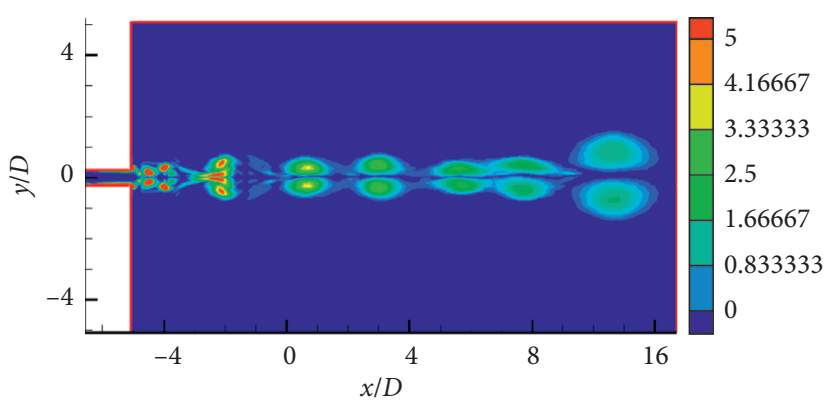

(b)

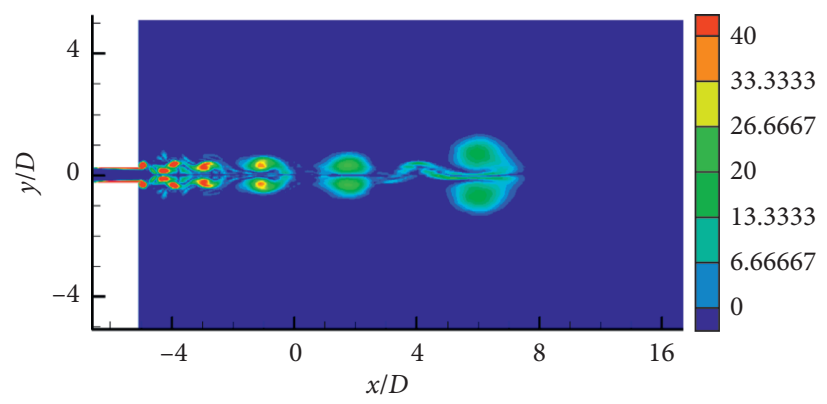

(c)

Figure 11: Vorticity field of synthetic jet at $S t=0.33$ : (a) $\operatorname{Re}=100$; (b) $\operatorname{Re}=1100$; (c) $\operatorname{Re}=2400$. 


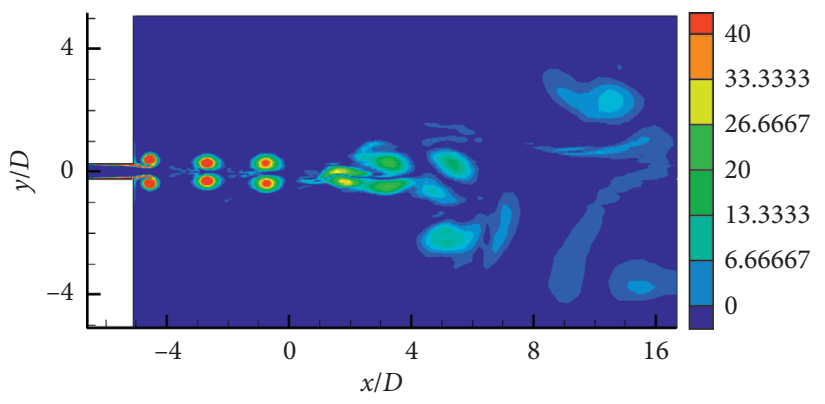

(a)

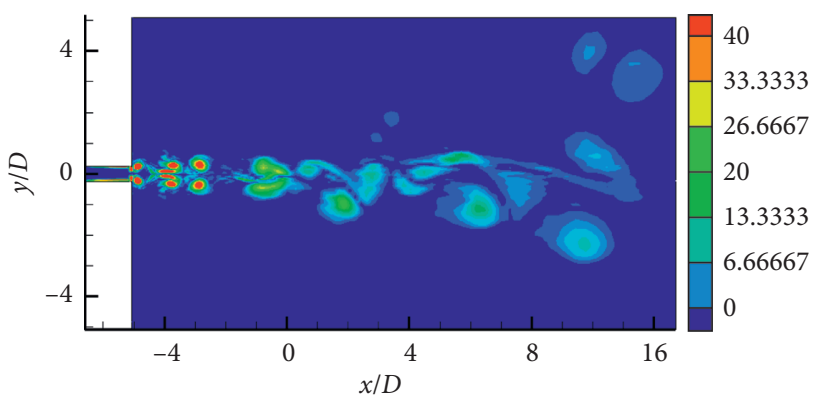

(b)

Figure 12: Fully developed synthetic jet under different Strouhal numbers at $\mathrm{Re}=2400$ : (a) $\mathrm{St}=0.11$; (b) $\mathrm{St}=0.33$.

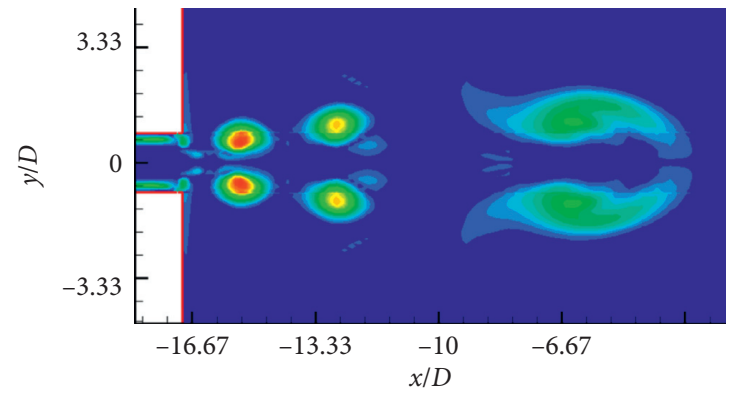

(a)

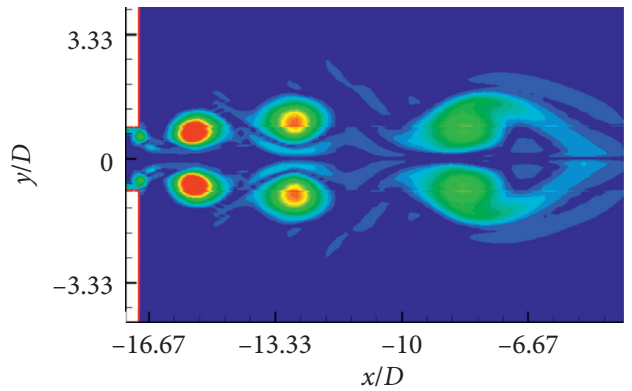

(b)

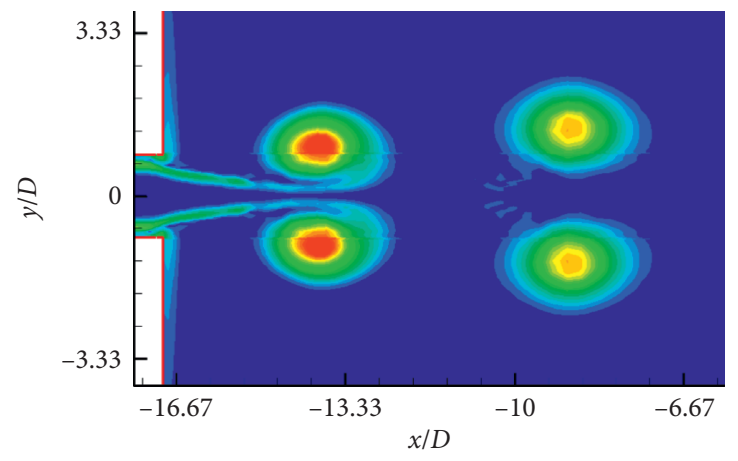

(c)

FiguRE 13: Vorticity fields of synthetic jet under different Reynolds numbers at $\mathrm{St}=0.22$ in near field: (a) $\mathrm{St}=0.22, \mathrm{Re}=1100$; (b) $\mathrm{St}=0.22$, $\operatorname{Re}=5918 ;$ (c) $\mathrm{St}=0.11, \mathrm{Re}=1100$.

adjacent vortex rings near the synthetic jet outlet is $d_{3}=5.0067 D$. In summary, the distance between two adjacent vortex rings near the exit of the synthetic jet goes hand in hand with the Strouhal number, independent of the Reynolds number.

\section{Conclusion}

In this manuscript, the numerical simulations of free jet and synthetic jet have been done in detail to analyze the vortex ring structures and flow characteristics of free jet and synthetic jet. The main conclusions are conducted as follows:

(1) Under the example simulated in this manuscript, starting from the Reynolds number $\mathrm{Re}=676$ does the secondary vortex get off in free jet vorticity field. When $\operatorname{Re}<676$, the shedding of the vortex rings does not happen in the vorticity field of free jet, and the flow field is in a relatively steady state. When the Reynolds number Re $>676$, the flow field of free jet is tending to lose stability due to the instability of the shear layer, and the shedding of the vortex rings may occur. As the Reynolds number goes up, the leading vortex and the secondary vortex of free jet will be merged together, and the vorticity and velocity of the vortex rings will ascend accordingly. The interaction between the secondary vortex and the leading vortex and the secondary vortex between one another will be more evident. This proves that the shedding and the fusion of these vortices have much to do with the Reynolds number.

(2) When under the condition of $\mathrm{Re}=100$ and the Strouhal number $S t=0.22$, the flow field structures of 
the synthetic jet are similar to that of free jet flow field at $\operatorname{Re}=100$. And the vortex rings of low vorticity intensity at the exit of the synthetic jet are soon absorbed by the nearby shear layer and jet. From the samples analyzed in this manuscript, a series of vortex rings are generated at the exit of the synthetic jet when $\operatorname{Re} \geq 500$. During the movement of the vortex ring towards downstream, the symmetry of the vortex ring retains in good state. And the vortex rings do not break up and are well arranged. With the values of the Reynolds number of the synthetic jet jumping up, there is a tendency for the vorticity field of the synthetic jet into turbulence.

(3) When $\mathrm{St}=0.11$, vortex rings are formed continuously in the vorticity field of the synthetic jet at $\mathrm{Re}=100$. However, there are no continuous vortex rings generated under the Strouhal number $\mathrm{St}=0.22$ and $\mathrm{St}=0.33$. When the Reynolds number is larger than 100, regardless of the value of the Strouhal number, vortex rings are unremittingly generated in the synthetic jet. When at the same Reynolds number, with the increase in the Strouhal number, the stability of the far field region of the vorticity field for synthetic jet will decrease unceasingly. The Strouhal number will affect the distance between the leading vortex and the trailing vortex and the trailing vortices between one another after the process of vortex ring fusion. And as the Strouhal number goes up, the distance of the two is dramatically reduced.

(4) The distance between two adjacent vortex rings near the synthetic jet exit is testified to have the close link between the Strouhal number, yet independent of the Reynolds number.

\section{Data Availability}

The data used to support the findings of this research are available from the corresponding author upon request.

\section{Conflicts of Interest}

The authors declare that there are no conflicts of interest regarding the publication of this paper.

\section{Acknowledgments}

The grant support from the National Natural Science Foundation of China (51909245), Science and Technology Innovation Projects of Higher Education in Shanxi Province (2019L0534), Aviation Science Foundation (2019ZA0U0001), and Natural Science Young Foundation of Shanxi China (nos. 201801D221234 and 201801D221210) are greatly acknowledged.

\section{References}

[1] P. Zhang, J. Wang, and L. Feng, "Research progress on zero mass jet technology and its application," Science in China (Series E), vol. 38, no. 3, pp. 321-349, 2008.
[2] S. Muppidi and K. Mahesh, "Study of trajectories of jets in crossflow using direct numerical simulations," Journal of Fluid Mechanics, vol. 530, pp. 81-100, 2015.

[3] C. Lee, G. Hong, Q. P. Ha, and S. G. Mallinson, "A piezoelectrically actuated micro synthetic jet for active flow control," Sensors and Actuators A: Physical, vol. 108, no. 1-3, pp. 168-174, 2003.

[4] L. L. Yuan, R. L. Street, and J. H. Ferziger, "Large-eddy simulations of a round jet in crossflow," Journal of Fluid Mechanics, vol. 379, pp. 71-104, 1999.

[5] M. Charmiyan, E. Azimian, F. C. Aloui, and D. Michaelis, "3D tomographic PIV, POD and vortex identification of turbulent slot jet flow impinging on a flat plate," Journal of Mechanical Science and Technology, vol. 31, no. 11, pp. 5347-5357, 2017.

[6] D. Degouet, B. P. M. W. van Esch, and M. Dubuisson, "Numerical and experimental investigation of tip leakage vortex trajectory and dynamics in an axial flow pump," Computers \& Fluids, vol. 112, pp. 61-71, 2015.

[7] F. F. Grinstein and C. R. Devore, "Dynamics of coherent structures and transition to turbulence in free square jets," Physics of Fluids, vol. 8, no. 5, pp. 1237-1251, 1996.

[8] R. B. Wicker and J. K. Eaton, "Near field of a coaxial jet with and without axial excitation," AIAA Journal, vol. 32, no. 3, pp. 542-546, 1994.

[9] J. Kim and H. Choi, "Large eddy simulation of a circular jet: effect of inflow conditions on the near field," Journal of Fluid Mechanics, vol. 620, pp. 383-411, 2009.

[10] S. A. Stanley, S. Sarkar, and J. P. Mellado, "A study of the flowfield evolution and mixing in a planar turbulent jet using direct numerical simulation," Journal of Fluid Mechanics, vol. 450, pp. 377-407, 2002.

[11] C. Bogey and C. Bailly, "Large eddy simulations of round free jets using explicit filtering with/without dynamic Smagorinsky model," International Journal of Heat and Fluid Flow, vol. 27, no. 4, pp. 603-610, 2006.

[12] J. W. Jewkes, A. King, and Y. M. Chung, "Large Eddy simulation of a steady circular jet issuing into quiescent fluid," in Proceedings of the 19th International Congress on Modelling and Simulation, Perth, Australia, December 2011.

[13] M. Gharib, E. Rambod, and K. Shariff, "A universal time scale for vortex ring formation," Journal of Fluid Mechanics, vol. 360, pp. 121-140, 1998.

[14] L. G. Pack and A. Seifert, "Periodic excitation for jet vectoring and enhanced spreading," Journal of Aircraft, vol. 38, no. 3, pp. 486-495, 2001.

[15] H. Yadav and A. Agrawal, "Effect of pulsation on the near flow field of a submerged water jet," Sādhanā, vol. 43, no. 3, p. 44, 2018.

[16] M. Amitay and F. Cannelle, "Evolution of finite span synthetic jets," Physics of Fluids, vol. 18, no. 5, Article ID 054101, 2006.

[17] H. Yadav, A. Agrawal, and A. Srivastava, "Mixing and entrainment characteristics of a pulse jet," International Journal of Heat and Fluid Flow, vol. 61, pp. 749-761, 2016.

[18] J. D. Holdeman and J. F. Foss, "The initiation, development, and decay of the secondary flow in a bounded jet," Journal of Fluids Engineering, vol. 93, no. 3, pp. 342-352, 1975.

[19] F. F. Grinstein, "Vortex dynamics and entrainment in rectangular free jets," Journal of Fluid Mechanics, vol. 437, pp. 69-101, 2001.

[20] J. Zhou, H. Tang, and S. Zhong, "Vortex roll-up criterion for synthetic jets," AIAA Journal, vol. 47, no. 5, pp. 1252-1262, 2009. 
[21] Z. Luo and Z. Xia, "Synthetic jet technology and its application in flow control," Advances in Mechanics, vol. 35, no. 2, pp. 221-234, 2005.

[22] X. Ma, Z. Zhang, and D. Yang, "Numerical study on the combined effect of two-dimensional synthetic jets," Journal of Aerodynamics, vol. 25, no. 4, pp. 509-512, 2007.

[23] B. E. Launder and D. B. Spalding, "The numerical computation of turbulent flows," Computer Methods in Applied Mechanics and Engineering, vol. 3, no. 2, pp. 269-289, 1974.

[24] J. J. Ai, S. C. M. Yu, A. W. K. Law, and L. P. Chua, "Vortex dynamics in starting square water jets," Physics of Fluids, vol. 17, no. 1, Article ID 014106, 2005.

[25] P. S. Krueger, J. O. Dabiri, and M. Gharib, "The formation number of vortex rings formed in uniform background coflow," Journal of Fluid Mechanics, vol. 556, pp. 147-166, 2006. 